\title{
Labyrinthe
}

20 | 2005 (1)

La Cognition

\section{Quelques questions posées au programme de sociologie cognitive}

Entretien avec Alban Bouvier

Philippe Lacour

\section{(2) OpenEdition}

\section{Journals}

Édition électronique

URL : http://journals.openedition.org/labyrinthe/751

DOI : $10.4000 /$ labyrinthe.751

ISSN : 1950-6031

Éditeur

Hermann

Édition imprimée

Date de publication : 20 avril 2005

Pagination : 135-154

\section{Référence électronique}

Philippe Lacour, «Quelques questions posées au programme de sociologie cognitive », Labyrinthe [En ligne], 20 | 2005 (1), mis en ligne le 10 juillet 2008, consulté le 10 décembre 2020. URL : http:// journals.openedition.org/labyrinthe/751 ; DOI : https://doi.org/10.4000/labyrinthe.751

Propriété intellectuelle 


\title{
QUELQUES QUESTIONS POSÉES AU PROGRAMME DE SOCIOLOGIE COGNITIVE
}

\author{
Entretien avec Alban Bouvier*
}

Alban Bouvier, agrégé de philosophie, est actuellement professeur à l'université d' Aix-Marseille-I après avoir été maître de conférences à la Sorbonne (Paris-IV, Département de philosophie et sociologie). Membre, pendant dix ans, du Groupe d'études des méthodes de l'analyse sociologique (CNRS), il fait actuellement partie de l'Institut Jean Nicod (CNRS/EHESS/ENS).

LABYRINTHE - Votre livre paru en 1995, L'Argumentation philosophique (Puf) était sous-titré Étude de sociologie cognitive. Plusieurs de vos articles portent un sous-titre analogue et vous accordez dans votre programme de recherche actuel une large place aux sciences cognitives. Vous diriez-vous pour autant cognitiviste et pourriez-vous nous indiquer, en tout état de cause, quelles sont les sciences cognitives que vous utilisez effectivement dans vos recherches et ce que vous pensez qu'elles apportent à votre réflexion?

Alban Bouvier - Ce qui m'intéresse directement dans les sciences cognitives pour mes propres recherches, c'est essentiellement la psychologie cognitive ou, plus exactement, un certain style de psychologie cognitive, la psychologie cognitive expérimentale. Il y a souvent, en effet, une confusion qui est faite entre deux, voire trois manières, pourtant au fond très différentes, de faire de la psychologie cognitive, à laquelle, en outre, ne se réduisent pas les sciences cognitives.

a) Le programme longtemps le plus visible en psychologie cognitive a été le programme dit «cognitiviste» ou encore computationnaliste, et ce deuxième qualificatif est nettement plus parlant car il indique

\footnotetext{
* Entretien électronique réalisé par Philippe Lacour.
} 
clairement le cour du programme en question: prendre les ordinateurs (computers) comme modèle de compréhension des processus cognitifs humains (ou, plus largement, animaux). Ce programme peut se moduler de façon plus complexe, vu les échanges possibles entre «modélisant» et «modélisé»: la neurophysiologie a ainsi suggéré à son tour un nouveau modèle d'ordinateurs, le modèle connexionniste (les connexions entre neurones servant à penser le fonctionnement de l'ordinateur «en réseau») qui, en retour, peut permettre de penser plus nettement le fonctionnement de l'esprit humain que la seule neurophysiologie descriptive. Mes propres recherches sont étrangères, pour l'essentiel, à ce programme cognitiviste ou computationnaliste dans ses différentes versions. Il m'intéresse cependant, les idées nées dans un programme pouvant émigrer dans un autre et le féconder. Il y a ainsi actuellement des recherches que je considère avec attention sans pourtant les utiliser directement et qui visent à simuler informatiquement les processus cognitifs sociaux, notamment la répartition (ou la « distribution») des connaissances (ou des informations) entre différents acteurs, ce qu'on appelle le modèle de la cognition distribuée.

b) Mais les recherches sur le cognitif ne se confondent pas avec le cognitivisme (comme on tend souvent à le penser). À côté de ces recherches et ne leur devant pour ainsi dire rien, il y a une psychologie cognitive expérimentale, dont les méthodes sont classiques. C'est elle que j'utilise au premier chef. Une bonne part des problèmes que cherche à résoudre cette psychologie vient de la théorie de la décision. Tversky et Kahneman, en particulier, ont mis systématiquement en évidence combien le comportement des acteurs était différent de ce que la théorie axiomatisée du choix rationnel pouvait dire: les gens font de grosses erreurs de raisonnement en fonction notamment de la manière dont les choix leur sont présentés (framing $)^{1}$; d'après une étude célèbre de Quattrone et Tversky, il semble aussi qu'il leur arrive souvent de confondre la cause et le signe d'une chose (sophisme dit « calviniste») ${ }^{2}$, etc. Ces travaux cherchent donc à identifier les biais de raisonnement les plus fréquents dans les choix les plus divers (prendre telle compa-

\footnotetext{
1. A. Tversky et D. Kahneman, «Judgment under Uncertainty: Heuristics and Biases », Science, 185, p. 1124-1131, 1974.

2. G. A. Quattrone \& A. Tversky, «Self-Deception and the voter's illusion », dans Elster, Jon (ed.), The Multiple Self, Cambridge University Press, 1986.
} 
gnie d'assurance plutôt que telle autre, voter ou ne pas voter, proposer tel programme de vaccination ou non, etc.). Je m'intéresse plus directement encore à des recherches davantage dispersées de psychologie expérimentale qui concernent les erreurs de raisonnement dans les croyances en dehors des seules procédures de décision (par exemple la psychologie des paralogismes dits de la fausse cause, la psychologie des paralogismes dits de composition et de division, etc.). Avant Frege et Russell, qui ont lutté contre le psychologisme en logique, tous les traités de logique comportaient une partie consacrée aux paralogismes et aux sophismes (le dernier grand traité de ce genre étant le Système de logique de Stuart Mill). Mais la psychologie, ce n'est pas forcément le psychologisme; or, à la suite des critiques de Frege et Russell (jointes à celles de Husserl), on a eu tendance à jeter le bébé avec l'eau du bain. Depuis deux ou trois décennies, la psychologie du raisonnement est revenue, en même temps qu'on a découvert que Stuart Mill n'était, de toute façon, pas aussi psychologiste qu'on l'avait cru; certains psychologues se sont alors mis explicitement sous sa bannière (Nisbet et Ross $^{3}$ ). Pour ma part, j'ai cherché à montrer dans divers articles que Vilfredo Pareto, un économiste-sociologue largement méconnu, s'était proposé de développer les conséquences en sociologie de la psychologie du raisonnement de $\mathrm{Mill}^{4}$. D'autres recherches, qui ne doivent rien non plus au modèle computationnaliste, mais qui relèvent de la psychologie expérimentale de la cognition infantile ont mis en évidence qu'il y a des catégories élémentaires universelles $\left(\mathrm{Keil}^{5}\right.$, Carey $\left.{ }^{6}\right)$. On peut émettre l'hypothèse que ces catégories (pas forcément innées mais se construisant très tôt par maturation et selon un processus à peu près identique quels que soient les contextes sociaux) offrent une résistance particulière aux modifications de croyances recherchées par une argumentation.

Mais j'ai surtout utilisé et j'utilise beaucoup encore la psychologie de la catégorisation ordinaire de E. Rosch, qui met en évidence qu'on ne catégorise pas spontanément à la manière aristotélicienne (ou bota-

\footnotetext{
3. R. Nisbett et L. Ross, Human Inference: Strategies and Shortcomings of Social Judgment, Englewood Cliffs, N.J., Prentice-Hall, 1980.

4. A. Bouvier, «Les paralogismes d'un point de vue sociologique », dans Hermès, ${ }^{\circ}$ 16, p. 45-55, 1995.

5. F. C. Keil, Concepts, Kinds, and Cognitive Development, Cambridge, MA, MIT Press, 1989.

6. S. Carey, Conceptual Change in Childhood, Cambridge, MA, MIT Press, 1985.
} 
nique ou zoologique) mais plutôt autour de «prototypes ${ }^{7} »$. La psychologie cognitive qui me semble essentielle en analyse d'argumentation inclut aussi la psychologie de l'attention et de la mémoire discursives; certains des travaux les plus intéressants sont déjà anciens mais sont perçus rétrospectivement comme relevant déjà de la psychologie cognitive expérimentale (Bartlett).

c) Au-delà, il est parfois utile de connaître les bases neurophysiologiques de ces diverses facultés, notamment pour étayer les hypothèses que l'on peut faire sur l'existence de processus cognitifs distincts (intervenant par exemple dans la compréhension des énoncés) en identifiant différents micro-processus neuropsychologiques plus ou moins autonomes («modules») constitutifs de ces facultés. La psychologie (cognitive) évolutionniste remonte encore plus haut en cherchant à expliquer l'émergence de ces processus par des mécanismes de sélection darwinienne ${ }^{8}$. Je m'écarte franchement, quant à moi, de l'idée que la voie royale actuellement en sciences sociales serait précisément de remonter jusqu'à ces hypothèses néo-darwiniennes, a fortiori d'en partir ${ }^{9}$.

LABYRINTHE - Le livre que vous avez publié il y a quelques années sur la Philosophie des sciences sociales était, quant à lui, sous-titré : Un point de vue argumentativiste en sciences sociales (Puf, 1999). Dans un article à paraître prochainement vous présentez à nouveau un programme "argumentativiste» en sociologie et en anthropologie ${ }^{10}$. Votre compréhension philosophique des sciences sociales implique ainsi une théorie de l'argumentation, mais, comme dans votre précédent livre, vous rapprochez pourtant résolument cette dernière de la cognition. Vous suggérez donc, si l'on prend au mot la vocation posi-

\footnotetext{
7. E. Rosch, « Principles of Categorization », dans E. Rosch and B. B. Lloyd (eds), Cognition and Categorization, p. 27-48, Hillsdale, NJ, Erlbaum, 1978.

8. Cette psychologie évolutionniste est parfois articulée au modèle computationnaliste (comme chez St. Pinker, Comment fonctionne l' esprit, Paris, Odile Jacob, 2000 [1997]), quoiqu'elle en soit logiquement indépendante (comme Fodor l'a récemment montré avec beaucoup de netteté, dans J. Fodor, The Mind Doesn't Work that Way, Cambridge, MIT Press, 2000).

9. C'est même l'axe essentiel d'un article récent: «Une démarche abductive, parcimonieuse et graduelle dans l'explication cognitive de la culture. L'exemple des croyances religieuses », à paraître en 2005 dans Denis Laborde (dir.), Faut-il en finir avec la notion de culture?

10. «Le programme argumentativiste en sociologie et en anthropologie cognitives », à paraître en 2005 dans Nicole Ramognino et Pierre Vergès, Sciences sociales et sciences cognitives.
} 
tive des sciences cognitives, que l'argumentation est susceptible d'être traitée scientifiquement. Mais, pourtant, l'argumentation ne requiertelle pas une analyse interprétative plus que scientifique?

ALBAN BOUVIER - Je vais répondre à votre question en plusieurs temps:

a) D'abord, vous semblez opposer interprétation et explication comme si ces modes d'intelligibilité étaient antinomiques. Votre opposition rappelle ainsi celle de Dilthey entre sciences de la nature et sciences de l'esprit et évoque plus généralement la tradition herméneutique, si ce n'est que chez Dilthey l'opposition n'était que méthodologique (et non pas ontologique, comme elle l'est le plus souvent chez les herméneutes). Mais je ne partage pas du tout cette idée. Je partage plutôt la position que certains, comme Raymond Boudon, prêtent parfois à Weber (de façon assez généreuse car Weber était beaucoup moins net), à savoir que la compréhension est un des modes de l'explication. Je dirais, plus précisément encore: la compréhension des raisons d'une action et donc des arguments qui sont avancés à l'appui de cette action fait a priori partie de l'explication. Cela ne veut évidemment pas dire que les raisons données à l'appui d'une action ou d'une croyance sont forcément les vraies causes de cette action ou de cette croyance, car on sait bien que parfois ces raisons sont des rationalisations post hoc, mais, si du moins les arguments sont vraisemblables, les raisons invoquées auraient pu être des causes de l'action ou de la croyance en question. La situation n'est donc pas épistémologiquement très différente d'une explication causale dans laquelle on peut invoquer par erreur (et éventuellement par malhonnêteté) une fausse cause.

b) Dans l'idéal, on peut souhaiter avoir une connaissance précise de ce qui se passe dans le cerveau des acteurs, mais cette connaissance fait actuellement défaut (on peut tout au plus repérer des différences de flux sanguin ou d'activité électrique dans certaines zones du cerveau, ce qui reste beaucoup trop macroscopique) et elle ne nous dirait, de toute façon, pas tout ${ }^{11}$. Dès lors, les moyens dont on dispose pour comparer des interprétations rivales des raisons d'une action ou d'une croyance et décider de celle qui est la plus probable sont seulement ceux du sens commun simplement rationalisés, précisés et raffinés. Une des méthodes les plus répandues en sciences sociales consiste

11. Voir infra, sixième question. 
ainsi à modéliser ces motivations possibles, en invoquant les motivations les plus vraisemblables ou les plus communes. Par exemple: les gens agissent plus fréquemment par intérêt que par devoir, par égoïsme que par altruisme. On peut raffiner sur ces principes (par exemple sur l'égoïsme) en prenant acte, par exemple, de ce qu'il est possible de prendre un certain plaisir au bonheur d'autrui ou, plus prosaïquement encore, qu'on peut avoir intérêt à la satisfaction d'autrui. On peut aussi invoquer des principes formels de cohérence psychologique : cohérence entre les arguments, cohérence entre ce qu'on dit croire et ce qu'on fait effectivement, etc. Différentes variantes de ces principes de méthode existent: principe de charité (version Quine ou version Davidson), principe de rationalité limitée (version Herbert Simon ou autre), etc. En un sens, on est bien dans le domaine de l'interprétation et la différence la plus patente avec l'interprétation des herméneutes (en dehors de l'opposition monisme/dualisme ontologique) est que l'on modélise: on se donne une représentation simplifiée des motivations possibles. Cela se justifie notamment lorsqu'on veut étudier les motivations d'acteurs sociaux en grand nombre à l'origine d'un phénomène social de très grande amplitude (l'attitude des consommateurs en matière de recherche d'un logement, d'acceptation d'une politique de santé publique, ou un processus macro-historique comme l'émergence du capitalisme, la construction de l'Europe, etc.) et que l'on a de bonnes raisons de penser que les différences de motivations peuvent être négligées au niveau de précision où l'on a décidé de se situer. En modélisant de cette façon la réalité, on reste toutefois encore fort éloigné de celle-ci. Si l'on veut s'approcher des raisons qui ont joué un rôle effectif dans l'émergence des phénomènes sociaux, il faut étudier les arguments effectivement employés par les acteurs pour justifier leurs choix ou ceux qui auraient pu être les leurs, même si ces arguments étaient d'intention fallacieuse.

c) Il y a souvent, en effet, une dimension normative dans les arguments que les acteurs sociaux donnent à l'appui d'une croyance ou d'une action: il y a les raisons (ou plus généralement les causes) pour lesquelles ils croient ou font quelque chose, et il y a les raisons pour lesquelles ils pensent qu'ils devraient croire ou faire quelque chose. Or, ce sont, bien entendu, souvent ces dernières qui sont invoquées par un acteur comme étant à la source de sa croyance ou de son action, même si l'acteur en question sait fort bien que ce ne sont pas les vrais argu- 
ments : pour prendre un exemple trivial, l'homme politique candidat à une fonction n'invoque souvent que son amour du bien public même quand il sait que ce qui le motive, c'est, au moins en partie, le goût du pouvoir et qu'il n'ignore nullement que les électeurs le soupçonnent précisément d'être fort probablement animé aussi par l'appétit du pouvoir. Ou, pour prendre un exemple moins trivial : lorsque les bourgeois et les banquiers des $\mathrm{XVII}^{\mathrm{e}}$ et $\mathrm{XVIII}^{\mathrm{e}}$ siècles ont voulu que l'interdit qui entourait le prêt à intérêt soit levé par l'Église catholique, ils n'ont pas invoqué les avantages financiers qu'ils en retireraient, qui étaient évidents; cet argument n'aurait eu, à lui seul au moins, aucun effet ou bien un effet exactement contraire à leur intention. Ils ont plutôt cherché à réfuter les arguments que les prêtres leur opposaient, à savoir de profiter de la misère de ceux qui empruntaient en faisant valoir qu'il y avait différents types d'emprunteurs, que certains n'étaient nullement pauvres et que, en outre, grâce à l'argent emprunté, l'emprunteur gagnerait beaucoup plus que ce qu'il devrait rendre en intérêts. Ils ont donc invoqué des normes d'ordre éthique et le cours de l'échange d'arguments laisse penser que ce sont ces arguments qui ont eu un rôle causal effectif sur la libéralisation progressive de l'éthique économique du catholicisme ${ }^{12}$.

D'autres normes sont d'ordre épistémologique. Ainsi, même si les mobiles les plus profonds de Pasteur contre la génération spontanée était sa foi catholique (et donc son «créationnisme»), les arguments dont il savait qu'ils étaient seuls épistémologiquement acceptables (et donc efficaces) dans la communauté scientifique et les seuls qu'il ait introduits dans ses publications scientifiques ont été d'ordre expérimental.

On se situe toujours ici au niveau interprétatif, c'est-à-dire au niveau de la description ou de la reconstruction du sens que les acteurs accordaient ou prétendaient accorder eux-mêmes à leurs actions ou à leurs croyances. Mais on dépasse ce niveau interprétatif lorsque, prenant acte de ce que des arguments valides ne convainquent pas ou bien sont mal compris et déformés, on cherche des causes au-delà des raisons, soit dans le contexte social, soit dans des processus cognitifs, par exemple biaisés. Je m'intéresse spécialement à ce second aspect, négligé dans les sciences sociales, tout en cherchant dans certaines études à exami-

12. Groethuysen, Les Origines de l'esprit bourgeois en France, Paris, Gallimard, 1927 [1926]. 
ner comment elles s'articulent aux premières ${ }^{13}$. Je suis ici toujours dans de l'explicatif mais qui n'est plus de l'interprétatif.

LABYRINTHE - Cette approche scientifique de l'argumentation vous permet-elle de conserver un trait caractéristique de la sociologie, en particulier, et des sciences sociales en général : la dimension critique? Vous parliez, dans l'introduction de votre Philosophie des sciences sociales, de penser "avec Habermas, contre Habermas»: l'aspect critique n' est-il pas ce qui vous sépare le plus du philosophe allemand?

ALBAN BOUVIER - Je vais répondre, là encore, en plusieurs temps:

a) Je ne crois pas, en effet, que la dimension critique soit inhérente aux sciences sociales. Bien entendu, en tant que citoyen et même comme personne humaine, bien des choses me révoltent. Mais je fais plutôt partie de ceux qui pensent qu'il est intellectuellement sain de séparer autant qu'il est possible science et critique éthique ou politique. Étudier les normes éthiques d'un groupe, ce n'est pas forcément porter un jugement sur elles ${ }^{14}$. Cette dernière tâche relève de ce que l'on appelle actuellement l'«éthique normative» et l'«éthique appliquée», elles-mêmes adossées à une "méta-éthique », et pour lesquelles j'ai un vif intérêt intellectuel.

b) Par ailleurs, en tant que citoyen, j'ai plutôt de la sympathie pour le type de critique sociale qu'on trouve dans la Théorie de l'agir communicationnel et, plus encore, dans les œuvres postérieures d'Habermas; j'apprécie notamment beaucoup la version rationaliste qu'il donne désormais de son «rousseauisme», à travers l'idée d'une «politique délibérative». J'ai, du reste, publié plusieurs textes sur la

13. A. Bouvier, « Modèles tocquevilliens en sociologie cognitive. Prédispositions sociales à forger et à accepter des arguments d'après Tocqueville », dans L'Acteur et ses raisons. Mélanges en l' honneur de Raymond Boudon, Paris, Puf, 2000.

14. La question se pose différemment au niveau épistémologique car si l'on peut concevoir une variation historique et culturelle dans la perception des normes logiques, on ne le peut, de l'avis général, dans les normes logiques elles-mêmes, alors que la question semble plus ouverte concernant les normes éthiques. De sorte qu'on peut à la fois étudier les normes logiques d'un groupe et porter un jugement sur elles, à la différence ce qui se passe en éthique, où les choses sont bien moins nettes. C'est pourquoi j'ai de plus en plus tendance à préférer caractériser mes propres recherches comme relevant de la social epistemology ou théorie sociale de la connaissance, laquelle inclut la référence nécessaire à des normes de connaissances dans l'étude de la dimension sociale du savoir, tout en se préoccupant, lorsqu'elle est «naturalisée» (comme chez Alvin Goldman, par exemple: A. Goldman, Knowledge in a Social World, Oxford, O.U.P., 1999), des processus effectifs de la connaissance, donc de psychologie et de sociologie cognitives. 
démocratie délibérative sous la forme participative que lui assigne Habermas. Dans ces textes, toutefois, je suis la même démarche scientifique que dans mes autres travaux; je cherche, par exemple, à analyser la dimension cognitive de ces formes de politiques (en l'occurrence les limites du gain cognitif souvent espéré), sans me livrer à la critique sociale ni même à la discussion des normes éthiques en tant que telles ${ }^{15}$.

c) Ce pour lequel je me sens le plus éloigné d'Habermas, en dehors du mélange des genres de la «science» et de la «critique», c'est sa façon de concevoir la théorie en sciences sociales, c'est-à-dire presque exclusivement par confrontation de théories. Bien entendu, la métathéorie comparative est légitime en sciences sociales et même spécialement utile en sociologie car il n'y a pas encore dans cette discipline de «science normale» (au sens de Kuhn), constituée en mainstream (comme en économie), de sorte qu'on puisse penser qu'il n'y a plus qu'à raffiner les principes et à développer leurs conséquences tout en cherchant à étendre leur champ d'application. La confrontation des grandes théories, notamment de celles des fondateurs qui avaient des idées très contrastées sur la voie que devait prendre la discipline y a donc encore un sens; je me suis livré moi-même à ce genre d'études, en montrant notamment l'apport inaperçu de Pareto pour formuler un programme original de sociologie cognitive ${ }^{16}$. Mais la théorie sociologique ne peut vraiment pas se réduire à confronter Marx, Weber, Durkheim, Mead et Parsons (ou Pareto). Il est essentiel actuellement de tenter de reconstruire la sociologie en tirant parti du tour beaucoup plus analytique qu'a pris l'économie. C'est ce qu'a fait James Coleman notamment dans ses magistrales Foundations of Social Theor $y^{17}$, encore peu connues du public français et dans lesquelles la part réservée à la dimension cognitive des phénomènes sociaux reste néanmoins fort réduite ${ }^{18}$. Vu les résistances que rencontre ce modèle et son impuissance à s'ériger en mainstream, il me semble utile de tenter des reconstructions plus complexes sur des fondements moins étroits, voire des

15. A. Bouvier, « Dimensions axiologique, épistémologique et cognitive de la délibération publique. Analyse d'un exemple », Cahiers d'économie politique, 2004, n 47, p. 215-234.

16. A. Bouvier (dir.), Pareto aujourd' hui, Paris, Puf, 1999.

17. Harvard, H.U.P., 1990.

18. Voir Revue française de sociologie, 2003, T. 44-2, n spécial, A. Bouvier et Ph. Steiner (éd.), $L a$

Théorie du choix rationnel: les Foundations of Social Theory de James S. Coleman en débat. 


\section{Labyrinthe, $n^{\circ} 20$}

reconstructions franchement alternatives, mais dans un style tout aussi analytique.

LABYRINTHE - On peut considérer que les notions d'innéité ou de nature humaine, sous-jacentes au programme cognitiviste, sont corrélées à l'idée que les processus essentiels se déroulent à une échelle temporelle qui échappe aux prises de la société et de la culture, et qui est tenue pour indispensable à l'étude des phénomènes humains. Ces notions ne ressortissent-elles pas à une forme de cécité "scalaire »qui fait croire que "one scale fits all», alors qu'aucune échelle n'est en réalité plus "réelle » qu' une autre ${ }^{19}$ ?

ALBAN BOUVIER - Il faut rappeler d'abord que les notions d'innéité ou de nature humaine non seulement ne sont pas inhérentes au programme de psychologie cognitive expérimentale, mais pas même au programme cognitiviste au sens strict de programme computationnaliste, et qu'elles ne sont intrinsèquement liées actuellement qu'au programme de psychologie évolutionniste.

D'autre part, il faut distinguer un programme, qui est un ensemble de préceptes de recherche, d'une doctrine, qui est un ensemble de thèses. Je n'ai méthodologiquement rigoureusement rien contre un programme qui ferait l'hypothèse que les processus essentiels se déroulent au niveau inné (mais rien non plus contre un programme symétrique). Parler de «cécité » me semble très excessif si l'on a en vue qu'il s'agit de «programme»: se mettre délibérément des «œillères » pour focaliser le regard dans une direction déterminée, ce n'est pas être aveugle... En revanche, je pense qu'il y a des phénomènes de seuils, de sorte que je crois qu'on ne peut en rester ni au niveau de ce qui est construit socialement, ni à celui des seuls processus biologiques et neurophysiologiques. Sur le fond, je suis d'accord avec l'idée qu'il faille distinguer différentes échelles temporelles et bien entendu d'accord aussi avec l'idée que l'étude des invariants cognitifs ne peut remplacer celle des variations culturelles et historiques. Mais mon sentiment général est que les études sur les invariants et sur les variations sont trop découplées et que l'on peut exprimer ce regret dans deux directions. Vous semblez surtout sensible au fait que ceux qui s'engagent dans les sciences cognitives sont trop indifférents aux variations

19. Voir dans ce dossier l'article de Jacques Plouin. 
historiques; mais on peut être sensible aussi au défaut symétrique: la relative indifférence des historiens, sociologues et ethnologues aux invariants. Cela me semble affaire et de dosage et de contexte (voire de micro-contexte) et il $\mathrm{y}$ a certainement un moment où on peut et même où on doit retirer ses œillères méthodologiques (quelles qu'elles soient) et élargir son champ de vision. Mais savoir quel est le moment propice, heuristiquement parlant, ne va pas toujours de soi. Pour ma part, dans un certain nombre d'articles échelonnés sur plusieurs années, j'ai cherché à montrer comment une approche centrée sur des invariants cognitifs pouvait éclairer des études d'ordre historique (par exemple la levée de l'interdit du prêt à intérêt au cours des $\mathrm{XVII}^{\mathrm{e}}$ et $\mathrm{XVIII}^{\mathrm{e}}$ siècles $^{20}$, le processus de rationalisation de l'Occident ${ }^{21}$, etc.); dans un article récent, j'ai plutôt voulu montrer en quelque sorte symétriquement comment le recours à des études centrées sur la recherche de processus innés déterminés par l'évolution n'était ni un requisit ni la meilleure méthode pour éclairer tel autre phénomène historique (la diffusion et la transformation des croyances religieuses), quoique je partage les mêmes convictions ontologiques et scientifiques générales que les auteurs de ces recherches évolutionnistes ${ }^{22}$.

Cela dit, l'opposition qui me semble la plus pertinente, et celle autour de laquelle j'organise mon travail méthodologiquement parlant, depuis quelques années, n'est de toute façon pas celle entre l'inné et l'acquis, ni celle entre le naturel et le social, ni entre l'invariant et le variable, mais entre le niveau de l' individuel et le niveau infra-individuel, que l'un et l'autre soient naturels ou culturels, innés ou acquis, etc. Je pense même qu'il faut ajouter la considération du point de vue des acteurs, lesquels sont souvent convaincus qu'il y a un niveau «holiste» (voir infra). Par ailleurs, une opposition qui me semble aussi capitale, c'est celle qu'il y a entre l'intentionnel (et donc conscient) et le mécanique (et donc non conscient) ${ }^{23}$ et qui fonde une part de la

20. A. Bouvier, «Argumentation et cognition en sociologie morale et juridique. Un exemple: les procédures de catégorisation dans le processus de légitimation du prêt à intérêt », dans R. Boudon, A. Bouvier, et F. Chazel, Cognition et Sciences sociales. La dimension cognitive dans l'analyse sociologique, Paris, Puf, 1997, 2 éd. 1999, traduit en italien chez Einaudi, Rome, 2000.

21. A. Bouvier, L'Argumentation philosophique, op. cit.

22. Voir note 9.

23. Le fonctionnement de l'attention et celui de la mémoire discursives dépendent peu de la volonté, par exemple; on est plutôt passif, à ce niveau, devant les stratégies rhétoriques de celui qu'on lit ou qu'on écoute: c'est lui qui cherche à fixer notre attention et à nous faire retenir telle ou telle idée. 
distinction entre l'explicatif interprétatif et l'explicatif non interprétatif (voir supra).

LABYRINTHE - Vous dites procéder selon une démarche "abductive». C'est-à-dire que, à l'opposé de la méthode évolutionniste appliquée aux sciences sociales, que vous considérez comme "déductive», car elle part des principes de la psychologie évolutionniste et examine quelles devraient être leurs implications en sciences sociales, vous partez des faits sociaux et vous cherchez leur explication en remontant, par voie régressive, vers les diverses théories possibles et déjà plus ou moins bien établies. Vous semblez donc reconnaître une certaine positivité aux faits sociaux, comme toute la tradition sociologique ${ }^{24}$. Cependant, vous jugez paradoxalement les deux méthodes "complémentaires", au moins "en principe», au nom d'un même naturalisme et d' un même matérialisme : n'y a-t-il pas contradiction à vouloir, d'un côté, autonomiser le social et, de l'autre, à reconnaître la pertinence de sa dissolution dans le psychologique?

Alban BOUVIER - Je prône effectivement une démarche plus «abductive», i.e. qui part des faits sociaux eux-mêmes parce que ce sont eux qu'il s'agit d'expliquer, mais aussi plus gradualisée parce que je pense qu'il existe des phénomènes de seuil (quand on passe d'un niveau à un autre) et qu'il n'est souvent ni nécessaire ni utile de remonter (par abduction) jusqu'au niveau des gènes, par exemple, pour expliquer nombre de phénomènes sociaux (ce que fait la psychologie évolutionniste à la manière de Pinker). Souvent l'explication pertinente au niveau des phénomènes sociaux est, au contraire, tout à fait neutre par rapport aux grands débats concernant le néo-darwinisme et l'adaptationnisme. Mais parfois aussi, les hypothèses néo-darwiniennes apportent quelque chose. Mon sentiment est seulement qu'à en partir on sollicite une grande machinerie explicative (avec son cortège de problèmes épistémologiques et méthodologiques propres) pour des résultats plutôt modestes.

Par ailleurs, concernant la positivité des faits sociaux, il y a manifestement malentendu entre nous. Je parle, du reste, le plus souvent de "phénomènes sociaux », pour éviter les connotations durkheimiennes de «faits sociaux». Je ne récuse bien entendu pas l'existence des faits

24. Voir aussi l'article de Jacques Plouin. 
sociaux quand on entend par là les effets agrégés de comportements individuels (par exemple la densité d'une population donnée, le protestantisme d'une population donnée, la division du travail, etc.). Mais je récuse absolument, en revanche, l'exigence d'assumer par là l'existence de phénomènes «collectifs » sui generis tels que la nation ou la famille; et pourtant je pense que Durkheim a eu une intuition très profonde, revitalisée actuellement par Margaret Gilbert, mais présente aussi chez certains philosophes allemands antérieurs à Durkheim, par exemple Herder ou encore Hegel, et qu'il faut intégrer cette intuition. Les uns et les autres sont en effet sensibles à ce que les acteurs sociaux ont le sentiment - et dans certains contextes plus que dans d'autres, par exemple dans les sociétés primitives ou dans la Cité grecque antique telle que Hegel la décrit au début du second tome de la Phénoménologie de l'esprit - que le groupe, la tribu, la Cité, la nation ont une existence indépendante d'eux, voire, en un sens, que leur existence comme individus est dérivée de l'existence de cette totalité. Il est vrai que parfois les acteurs sociaux se sentent non simplement individus mais aussi membres d'un groupe et éventuellement membres d'un groupe (comme citoyens par exemple) avant que d'être un individu (ou une personne). Cette sensibilité intellectuelle est au fondement du holisme méthodologique en ce qu'il a de légitime et qui consiste à prendre acte de ce vécu, voire à en partir. Ce que je récuse absolument, c'est seulement que le chercheur ait à assumer ce réalisme des phénomènes collectifs (cette ontologie holiste), comme le fait très explicitement Durkheim. Certains philosophes sociaux, comme le Finlandais Raimo Tuomela, ont cherché à articuler cette forme de holisme méthodologique (mais non ontologique) avec l'individualisme méthodologique ${ }^{25}$. Ce que je reprocherai éventuellement à la perspective actuelle de Dan Sperber ${ }^{26}$ et de Pascal Boyer ${ }^{27}$, c'est précisément de ne pas intégrer vraiment ce point de vue, même si, je crois, ils n'auraient aucune objection de principe à ce genre de perspective, Sperber distinguant notamment très clairement «ontologie savante» et «ontologie indigène».

25. R. Tuomela, The Importance of Us. A Philosophical Study of Basic Social Notions, Stanford, Stanford University Press, 1995.

26. La Contagion des idées. Théorie naturaliste de la culture. Paris, Odile Jacob, 1996.

27. The Naturalness of Religious Ideas. A Cognitive Theory of Religion, Berkeley, University of California Press, 1994. 
LABYRINTHE - Vous invitez vous-même à la «prudence» dans la voie régressive menant des faits sociaux à la psychologie. Mais témérité et prudence se fondent sur une même position ontologique qui n'accorde de "réalité » qu' aux états de choses, au détriment du symbolique, dont François Rastier souligne au contraire à bon droit la positivité, la dignité ontologique. L'existence d' une sphère symbolique ne constituet-elle pas précisément une sorte de cran d'arrêt à une réduction matérialiste? Sinon comment, par quel critère, pouvez-vous décider de la "parcimonie» que vous prônez, et selon laquelle on ne saurait remonter trop haut dans l'explication des phénomènes: ne court-on pas le risque de l'arbitraire? C'est ce problème que vous abordez lorsque vous dites que, sans défendre un discontinuisme métaphysique, il faut cependant prêter attention aux phénomènes de seuil: mais, de nouveau, comment justifier alors votre "gradualisme»?

AlbAN BOUVIER - Je veux bien a priori reconnaître une «dignité ontologique» au symbolique, mais je craindrais un malentendu car, comme disait Aristote, «exister» se dit en plusieurs sens. Même si l'on adopte une position matérialiste et que l'on pense donc que le support des idées est et n'est que cérébral (des neurones et de la matière gliale), cela heurte l'intuition de dire que les idées elles-mêmes sont «matérielles »; on garde la sensation intellectuelle très forte que le type d'être des idées doit être différent de celui des neurones puisqu'on ne s'attend pas à les observer au bout d'un microscope!... Sans pour autant renoncer en rien au pur matérialisme, on peut marquer cette réticence par une distinction terminologique élégante due à Meinong (dans un autre cadre) et dire, par exemple, qu'elles «subsistent » plutôt qu'elles n' « existent» et ajouter, pour utiliser cette fois une notion introduite en philosophie de l'esprit et, me semble-t-il, assez heureuse en l'occasion, qu'elles «surviennent» sur la matière cérébrale. J'en tire qu'il n'est pas nécessaire de supposer, de façon mystérieuse et ontologiquement très dispendieuse, un monde d'idées existant par elles-mêmes, de manière transcendante aux individus. Je crois qu'on peut avoir un point de vue similaire pour ce qui est de la signification. Si l'on parle du mode d'être des énoncés linguistiques (et non du mode d'être de leur sens), on doit reconnaître aisément que ce ne sont que des traces graphiques ou des traces sonores: ces traces existent donc au sens matériel le plus banal. Mais dans tel groupe ou telle communauté donnée, telle séquence de ces traces sera considérée comme un ensemble de « signes » renvoyant 
à un sens visé par le producteur des traces en question. On pourra alors dire de ce sens qu'il «subsiste» dans l'esprit du producteur et qu'un sens sera reconstruit dans l'esprit de chacun des «récepteurs» de ces signes, sens qui « subsistera » dans leur esprit. On n'a donc pas à supposer non plus ici un monde transcendant, en l'occurrence un monde de significations.

Mais derrière la revendication par François Rastier d'une dignité ontologique et donc a fortiori d'une spécificité ontologique du symbolique (c'est-à-dire des signes doués de sens ou - plus exactement, je suppose - du sens lui-même), il y a bien entendu la revendication de la spécificité d'une discipline, la linguistique, comme c'était le cas chez Durkheim à propos de la sociologie. Rastier et, en général, les linguistes structuralistes français, me semblent soulever un véritable problème. Certains linguistes semblent en effet vouloir se contenter de faire de la psycholinguistique, dont l'objet est d'étudier comment se forgent (et se reçoivent) dans l'esprit les énoncés, et associer celle-ci à une psychologie cognitive plus générale examinant les opérations intellectuelles mises en jeu. La linguistique chomskyenne, la linguistique cognitive sont des variations sur cette idée ${ }^{28}$. On pourrait, à vrai dire, «socialiser » cette psycholinguistique en faisant remarquer que l'enfant apprend une langue dans des interactions avec son environnement, montrer encore que cet environnement est variable et faire alors une véritable sociolinguistique. Mais ce que les structuralistes ont en vue est encore différent: tout ce qui précède n'est pas encore suffisant à leurs yeux et ne touche en rien à l'essentiel. Manifestement, en effet, il est possible de laisser de côté les locuteurs - et donc les producteurs d'énoncés -, au moins provisoirement (toujours les œillères méthodologiques) et de comparer ces énoncés entre eux, d'étudier les relations des éléments mêmes de ces énoncés entre eux, etc. (les sèmes ou/et les sémèmes, ainsi que les éléments matériels sonores ou/et graphiques porteurs de ces éléments de sens, tels les phonèmes). Cet objet-là est l'objet d'études spécifiques des linguistes structuralistes (la langue, par opposition à la parole). Mais cet objet, telle langue donnée ou telle autre, n'a d'autre existence que dans l'esprit des linguistes (et dans celui des

28. Chomsky a associé de manière très forte cette psycholinguistique et cette psychologie cognitive à une thèse innéiste; mais, comme je l'ai fait remarquer plus haut, il n'y a pas de lien logique nécessaire entre ces programmes et cette thèse (a fortiori avec le néo-darwinisme). 
lecteurs d'ouvrages de linguistique). Le commun des mortels n'en possède en son esprit au mieux que des bribes, par les cours de grammaire et la consultation épisodique des dictionnaires, quand il a ou a eu accès aux uns ou aux autres. On peut, bien entendu aussi, étendre cette conception structurale à des signes qui ne sont pas linguistiques: telle figure, par exemple un dragon, peut être considérée, dans telle culture, comme renvoyant à un sens déterminé (par opposition et différence avec d'autres animaux, fabuleux ou non, dans la même culture, selon le modèle proprement structuraliste), sens éventuellement spécifié par le contexte particulier de l'énonciation ${ }^{29}$. Je ne nie nullement la légitimité et l'intérêt de ce genre d'étude.

Mais je veux surtout marquer que cette question n'a rien à voir avec le phénomène de seuil reconnu plus haut et donc rien à voir avec la défense de l'idée d'une spécificité proprement ontologique de la langue. En effet, reconnaître la légitimité d'une étude de la langue au sens que je viens de dégager, cela n'a encore rien à voir avec le holisme ontologique au sens de la sociologie et de la philosophie sociale (et dont j'ai parlé précédemment), lequel consisterait à penser, tel Herder, qu'il y a un «esprit de la langue» irréductible à toute analyse (de même qu'il y aurait un «esprit de la culture» ou un «esprit d'un peuple»). C'est bien sûr le sentiment que les locuteurs d'une langue ont souvent de leur propre langue et éventuellement d'une langue étrangère qu'ils peinent à maîtriser complètement. Et probablement faut-il aussi en tenir compte. Mais les structuralistes n'ont jamais soutenu une telle idée, foncièrement romantique au bout du compte. Or, c'est l'adhésion seule à cette forme de romantisme qui obligerait, me semble-t-il, à reconnaître une existence sui generis et donc une dignité ontologique particulière à la langue, comme Hegel à la Cité grecque et Durkheim à la nation moderne.

LABYRINTHE - Vous cherchez à justifier votre démarche au nom du nominalisme et de l'individualisme méthodologique. Mais il vous faut pour cela déceler chez les psychologues évolutionnistes eux-mêmes une forme de nominalisme : comment parvenez-vous à étayer cette lecture?

29. Le sens d'un mythe ou d'un conte, comme y a insisté Sperber, ne se dégage pas par la simple analyse de son sens symbolique au sein d'une culture donnée, pas plus que celui d'un énoncé, mais rapporté au contexte particulier de l'énonciation de ce mythe ou de ce conte, comme pour un énoncé. 
Par ailleurs, comment pouvez-vous concilier l'invocation de mécanismes cognitifs qui, comme vous le reconnaissez, impliquent toujours l'infra-individualisme, avec un individualisme méthodologique avoué?

ALBAN BOUVIER - Le nominalisme est adopté de façon très explicite, parmi ceux qui se réclament de l'évolutionnisme, par Dan Sperber. Dans certains textes, lorsqu'il critique la prééminence, voire l'exclusivisme, de l'individualisme méthodologique (désormais IM) et donc de l'individu comme niveau d'analyse, il est assez caustique par rapport à l' $\mathrm{IM}^{30}$. Dans d'autres, lorsqu'il reconnaît, au moins en principe, la légitimité de ce niveau d'analyse, il l'est moins ${ }^{31}$. Lui ainsi que Pascal Boyer et, de façon beaucoup plus polémique, Tooby et Cosmidès ${ }^{32}$, etc., critiquent bien davantage les conceptions holistes (au sens de Durkheim et de Hegel) ${ }^{33}$. L'individualisme méthodologique et l'infra-individualisme méthodologique partagent donc la critique nominaliste des entités ontologiques collectives et des concepts abstraits qui les véhiculent subrepticement. L'adoption de l'hypothèse particulière de la modularité de l'esprit (par Sperber par exemple), autrement dit de l'existence de mécanismes cognitifs spécialisés et relativement autonomes, implique, certes, la reconnaissance de l'infra-individualisme comme programme mais elle n'exclut pas, en principe, l'idée qu'il y ait des processus unificateurs (des «systèmes centraux », comme on dit dans cette littérature) au niveau de l'individu, éventuellement inconscients, justifiant qu'on prenne celui-ci comme atome provisoire de l'analyse (c'est-à-dire qu'on pratique l'IM). Il est vrai que l'individualisme méthodologique a été historiquement lié de fait à un intentionnalisme, mais celui-ci n'est pourtant pas logiquement impliqué par la règle de

\footnotetext{
30. D. Sperber, «Les sciences cognitives, les sciences sociales et le matérialisme», dans D. Andler (dir.), 1992, Introduction aux sciences cognitives, Paris, Gallimard, p. 397-420.

31. D. Sperber, «Individualisme méthodologique et cognitivisme », dans R. Boudon, A. Bouvier, F. Chazel (dir.), Cognition et sciences sociales, Paris, Puf, 1997, p. 123-136.

32. J. Tooby et L. Cosmides, «The Psychological Foundations of Culture », dans J. Barkow, L. Cosmides, J. Tooby, The Adapted Mind. Evolutionary Psychology and the Generation of Culture, Oxford, O.U.P., 1992, p. 19-136.

33. Ce qu'ils critiquent aussi - mais ce sont deux critiques logiquement indépendantes de la précédente -, c'est la tentation de la linguistique structurale et de la sémiotique (la science générale des signes) structurale en général de rejeter la légitimité de l'approche psycholinguistique ou psychocognitive. Cette critique est par ailleurs logiquement indépendante, quoique souvent confondue avec elle, d'un troisième type de critique, présent chez D. Sperber et D. Wilson, et d'inspiration proprement pragmatique ou contextualiste à l'encontre du modèle sémiotique (voir note 27).
} 
l'IM, laquelle demande uniquement que l'on remonte toujours aux individus pour expliquer les phénomènes sociaux; cette exigence de toujours fonder les phénomènes macrosociologiques (ou macro-économiques) sur du microsociologique (respectivement: micro-économique) n'implique pas comme telle l'exigence supplémentaire éventuelle de remonter aux intentions des individus.

LABYRINTHE - Vos travaux cherchent à montrer la pertinence d'une analyse des mécanismes généraux, concernant les croyances, par opposition aux analyses gilbertiennes, que vous considérez comme trop entachées de phénoménologie. Mais ne tirez-vous pas alors les sciences sociales du côté de la seule généralisation, à rebours de l'idée de Max Weber, pour qui la généralisation n'est qu'un outil pour penser une singularité historique, comme le souligne à son tour Jean-Claude Passeron?

AlbAN BOUVIER - Là encore, je distinguerais plusieurs questions.

D'abord, je ne considère pas que les analyses gilbertiennes soient «trop » entachées de phénoménologie. Je considère plutôt que Margaret Gilbert $^{34}$ en reste à l'analyse phénoménologique, alors que l'on a besoin, en outre, d'une analyse explicative des mécanismes sousjacents aux processus qu'elle décrit, au demeurant de façon exemplaire. Par ailleurs, les analyses de Gilbert ont beau être phénoménologiques, donc descriptives, elles ne décrivent encore que la forme générale et stylisée de certaines expériences : le fait de se promener avec quelqu'un sur un campus par exemple, mais pas telle promenade particulière de tel personnage historique particulier avec tel autre... De façon générale, Gilbert ne prend que très rarement et très allusivement des exemples historiques précis et donc vraiment singuliers, ce que j'essaie de faire régulièrement et presque systématiquement.

Assurément, Max Weber analyse bien, quant à lui, des phénomènes singuliers et il les analyse essentiellement dans leur devenir, faisant donc œuvre d'historien. Mais Weber ne cherche pas seulement à décrire une succession de phénomènes singuliers, comme vous tendez, me semble-t-il, à le suggérer. Il utilise des concepts «modélisant» cette succession, des modèles qui ont donc, par nature, un certain niveau de

34. M. Gilbert, On Social Facts, Princeton, Princeton University Press, 1989; M. Gilbert, Marcher ensemble. Essais sur les fondements des phénomènes collectifs, Paris, Puf, 2003. 
généralité et sont, pour cette raison, exportables sur d'autres objets. Le modèle de ce que Raymond Boudon appelle des «effets de composition simples », type particulier d'effets paradoxaux ou contre-intentionnels, joue ainsi le rôle clé pour expliquer le rôle du protestantisme dans l'émergence du capitalisme ${ }^{35}$, même si Weber n'a dégagé explicitement le rôle théorique majeur de ce modèle ni dans L'Éthique protestante, ni dans Économie et Société, ni même dans les Essais sur la théorie de la science.

Dans ce cas particulier (mais non toujours), Weber a donc péché, à mon goût, par défaut d'abstraction sur une question essentielle. Mais Weber pratique, en outre, de l'histoire explicative. Comme Tocqueville à propos de la révolution française ou Sombart à propos de l'absence de socialisme au début du $\mathrm{XX}^{\mathrm{e}}$ siècle aux États-Unis, il cherche à dégager, derrière la simple succession des faits, un enchaînement de causes et d'effets ${ }^{36}$. Weber utilise ainsi une hypothèse générale explicative forte, à savoir que les croyances religieuses jouent un rôle dans l'émergence de formes économiques spécifiques, en vertu notamment de l'éthique économique particulière qu'elles comportent chacune et qui produit, indépendamment de la conscience et de l'intention des individus, un ethos (une manière d'être) particulier. Weber est toutefois loin de mettre systématiquement à l'épreuve cette hypothèse (par une succession de vérifications/falsifications), à la manière, par exemple, du Durkheim du Suicide. Weber pèche ici par manque de rigueur inductive ${ }^{37}$.

Que le but de la théorie en sciences sociales, ce soit d'expliquer des faits, forcément singuliers, je suis prêt à l'accorder à Jean-Claude Passeron, mais il faut le dire aussi des sciences de la nature comme des sciences sociales qui, de mon point de vue, n'ont pas des méthodes foncièrement différentes. La théorie astronomique, la théorie géolo-

35. Le capitalisme est dû notamment, pour Weber, à une simple addition de comportements individuels (travailler autant qu'on peut sans profiter des fruits de son travail) aux conséquences paradoxales (épargner de l'argent et pouvoir investir et réinvestir). D'autres effets paradoxaux («effets de composition complexes ») s'expliquent par les interférences avec les actions des autres. Menger et Pareto les ont bien identifiés.

36. Il s'agit, en l'occurrence, essentiellement d'histoire économique (comme Weber en a revendiqué la désignation) ou d'histoire religieuse, selon que l'on considère l'effet ou (respectivement) la cause. 37. La sociologie cognitive, au sens où je l'entends, chercherait plus spécialement à expliquer par quels processus cognitifs les arguments des pasteurs luthériens et calvinistes (et, au premier chef, la dimension argumentative spécifique de la traduction luthérienne de la Bible) ont pu produire un tel ethos. 


\section{Labyrinthe, $n^{\circ} 20$}

gique, la théorie climatologique étudient aussi des faits singuliers (les révolutions de Mercure, l'éruption du Krakatoa, le phénomène El Niño de 1998 ou celui de 1999, etc.) avec la perspective de connaître d'autres faits singuliers; mais pour cela les hypothèses générales et les modélisations intermédiaires sont nécessaires. Sur ce plan, je m'écarte donc de Jean-Claude Passeron. 